\title{
Anisotropic Charged Fluid Sphere in Isotropic Coordinates
}

\author{
Neeraj Pant, ${ }^{1}$ N. Pradhan, ${ }^{2}$ and Ksh. Newton Singh ${ }^{2}$ \\ ${ }^{1}$ Mathematics Department, National Defence Academy, Khadakwasla, Pune 411023, India \\ ${ }^{2}$ Physics Department, National Defence Academy, Khadakwasla, Pune 411023, India \\ Correspondence should be addressed to Neeraj Pant; neeraj.pant@yahoo.com
}

Received 8 July 2014; Revised 22 August 2014; Accepted 22 August 2014; Published 2 September 2014

Academic Editor: Sergey D. Odintsov

Copyright (C) 2014 Neeraj Pant et al. This is an open access article distributed under the Creative Commons Attribution License, which permits unrestricted use, distribution, and reproduction in any medium, provided the original work is properly cited.

\begin{abstract}
We have presented a class of charged superdense star models, starting with a static spherically symmetric metric in isotropic coordinates for anisotropic fluid by considering Hajj-Boutros-(1986) type metric potential and a specific choice of electrical intensity $E$ and anisotropy factor $\Delta$ which involve charge parameter $K$ and anisotropy parameter $\alpha$. The solution is well behaved for all the values of Schwarzschild compactness parameter $u$ lying in the range $0<u \leq 0.2086$, for all values of charge parameter $K$ lying in the range $0.04 \leq K \leq 0.111$, and for all values of anisotropy parameter $\alpha$ lying in the range $0.016 \geq \alpha \geq 0$. With the increase in $\alpha$, the values of $K$ and $u$ decrease. Further, we have constructed a superdense star model with all degree of suitability. The solution so obtained is utilized to construct the models for superdense star like neutron stars $\rho_{b}=2.7 \times 10^{14} \mathrm{~g} / \mathrm{cm}^{3}$ and strange quark stars $\rho_{b}=4.6888 \times 10^{14} \mathrm{~g} / \mathrm{cm}^{3}$. For $K=0.06$ and $\alpha=0.01$, the maximum mass of neutron star is observed as $M=1.53 \mathrm{M}_{\odot}$ and radius $R=11.48 \mathrm{~km}$. Further for strange quark stars $M=1.16 \mathrm{M}_{\odot}$ and $R=8.71 \mathrm{~km}$ are obtained.
\end{abstract}

\section{Introduction}

Since the formulation of Einstein-Maxwell field equations, the relativists have been proposing different models of immensely gravitating astrophysical objects by considering the distinct nature of matter or radiation (energy-momentum tensor) present in them. Einstein-Maxwell field equations with anisotropic matter in isotropic coordinates have more importance over Einstein field equations for perfect fluid in curvature coordinates due to following rationale justifications.

(i) The presence of some charge may avert the catastrophic gravitational collapse by counterbalancing the gravitational attraction by the electric repulsion in addition to the pressure gradient.

(ii) The inclusion of charge inhibits the growth of space time curvature which has a great role in avoiding singularities (Ivanov [1]; de Felice et al. [2]).

(iii) Bonnor [3] pointed out that a dust distribution of arbitrarily large mass and small radius can remain in equilibrium against the pull of gravity by a repulsive force produced by a small amount of charge. (iv) The solutions of Einstein-Maxwell equations are useful to study the cosmic matter.

(v) The charge dust models and electromagnetic mass models are providing some clue about the structure of electron (Bijalwan [4]) and Lepton model (Kiess [5]).

(vi) Several solutions which do not satisfy some or all the conditions for well-behaved nature can be renewed into well-behaved nature by charging them.

(vii) Maharaj-Takisa [6] pointed out that the astrophysical objects have essential characteristics of rotational motion, which is caused by the presence of anisotropic parameter. Therefore, the matter in reality cannot be perfect fluid; Ruderman [7] and Sharma and Maharaj [8] also justified that the anisotropic always prevails in a certain density range $\approx 10^{15} \mathrm{~g} / \mathrm{cm}^{3}$.

(viii) Ivanov [9] pointed out that solutions in isotropic coordinates are more significant than the solutions in curvature coordinates, due to the following reasons: (a) the solutions in isotropic coordinates are simple in terms of algebraic expressions; (b) isotropic coordinates solutions can be used as seed solutions in Quasar modeling or nonstatic solutions. 
Thus for realistic model it is desirable to study the insinuation of Einstein-Maxwell field equations with reference to the general relativistic prediction of gravitational collapse with anisotropic matter. For this purpose charged fluid ball models are required. The external field of such ball is to be matched with Reissner-Nordstrom solution. The solutions of EinsteinMaxwell field equations successfully explain the characteristics of massive objects like neutron star, quark star, or other superdense objects. Further, these stars are specified in terms of their masses and densities.

(a) A neutron star has surface density $\rho_{b}=2.7 \times$ $10^{14} \mathrm{~g} / \mathrm{cm}^{3}$ (Astashenok [10]) and mass $1.4 \mathrm{M}_{\odot}-$ $2.9 \mathrm{M}_{\odot}$. However, Astashenok [10] established that $f(R)$ models with realistic equation of state of neutron star have upper limit mass $2 \mathrm{M}_{\odot}$ and minimal radius close to $9 \mathrm{~km}$.

(b) A strange quark star has surface density $\rho_{b}=4.6888 \times$ $10^{14} \mathrm{~g} / \mathrm{cm}^{3}$ (Fatema and Murad [11]; Zdunik [12]) and possible maximum mass $2 \mathrm{M}_{\odot}$. However, Dong et al. [13] established that due to presence of half skyrmions in the dense baryonic matter the stable strange quark star can have upper mass limit up to $2.4 \mathrm{M}_{\odot}$.

In recent past, a considerable number of exact solutions with well-behaved nature of general relativistic field equation with anisotropic matter have been obtained; Dev and Gleiser [14], Komathiraj and Maharaj $[15,16]$, Thirukkanesh and Regel [17], Takisa and Maharaj [18, 19], Mak and Harko [20], Mak et al. [21], Ivanov [1], Maurya and Gupta [22, 23], Chaisi and Maharaj [24], and Feroze and Siddiqui [25] deal with curvature coordinates and some of them are charged models. By motivation of Maharaj-Takisa [6] and Ivanov [9], in this paper, we present a new class of well-behaved exact solutions of Einstein-Maxwell field equations in isotropic coordinates for anisotropic fluid assuming a particular form of one of the metric potentials and suitable choice of electric intensity and anisotropy.

\section{Field Equations in Isotropic Coordinates}

We consider the static and spherically symmetric metric in isotropic coordinates

$$
d s^{2}=-e^{\omega}\left[d r^{2}+r^{2}\left(d \theta^{2}+\sin ^{2} \theta d \phi^{2}\right)\right]+e^{\nu} c^{2} d t^{2},
$$

where $\omega$ and $v$ are functions of $r$.

The Einstein-Maxwell field equations for a nonempty space-time are

$$
\begin{aligned}
R_{j}^{i}-\frac{1}{2} \delta_{j}^{i} R=-\frac{8 \pi G}{c^{4}} T_{j}^{i} & \\
=-\frac{8 \pi G}{c^{4}} & {\left[\left(p_{\perp}+\rho c^{2}\right) v^{i} v_{j}-p_{\perp} \delta_{j}^{i}\right.} \\
& +\left(p_{r}-p_{\perp}\right) \chi_{j} \chi^{i} \\
& \left.+\frac{1}{4 \pi}\left(-F^{i m} F_{j m}+\frac{1}{4} \delta_{j}^{i} F_{m n} F^{n m}\right)\right],
\end{aligned}
$$

where $R_{j}^{i}$ is Ricci tensor, $T_{j}^{i}$ is energy-momentum tensor, $R$ is the scalar curvature, $F_{j m}$ is the electromagnetic field tensor, $p_{r}$ denotes the radial pressure, $p_{\perp}$ is the transversal pressure, $\rho$ is the density distribution, $\chi^{i}$ is the unit space-like vector in the radial direction, and $v^{i}$ is the velocity vector, satisfying the relation

$$
g_{i j} v^{i} v^{j}=1
$$

Since the field is static,

$$
v^{1}=v^{2}=v^{3}=0, \quad v^{4}=\frac{1}{\sqrt{g_{44}}} .
$$

Thus we find that for the metric (1) under these conditions and for matter distributions with anisotropic pressure the field equation (2) reduces to the following:

$$
\begin{gathered}
\frac{8 \pi G}{c^{4}} p_{r}=e^{-\omega}\left[\frac{\omega^{\prime 2}}{4}+\frac{\omega^{\prime}}{r}+\frac{\omega^{\prime} \nu^{\prime}}{2}+\frac{\nu^{\prime}}{r}\right]+\frac{q^{2}}{r^{4}} \\
\frac{8 \pi G}{c^{4}} p_{\perp}=e^{-\omega}\left[\frac{\omega^{\prime \prime}}{2}+\frac{\nu^{\prime \prime}}{2}+\frac{\nu^{\prime 2}}{4}+\frac{\omega^{\prime}}{2 r}+\frac{\nu^{\prime}}{2 r}\right]-\frac{q^{2}}{r^{4}} \\
\frac{8 \pi G}{c^{2}} \rho=-e^{-\omega}\left[\omega^{\prime \prime}+\frac{\omega^{\prime^{2}}}{4}+\frac{2 \omega^{\prime}}{r}\right]-\frac{q^{2}}{r^{4}},
\end{gathered}
$$

where prime $\left(^{\prime}\right)$ denotes differentiation with respect to $r$. From (5) and (6) we obtain the following differential equation in $\omega$ and $v$ :

$$
\begin{aligned}
& e^{-\omega}\left[\frac{\omega^{\prime \prime}}{2}+\frac{v^{\prime \prime}}{2}+\frac{\left(v^{\prime}\right)^{2}}{4}-\frac{\left(\omega^{\prime}\right)^{2}}{4}-\frac{\omega^{\prime} \cdot v^{\prime}}{2}-\frac{\omega^{\prime}}{2 r}-\frac{v^{\prime}}{2 r}\right]-2 \frac{q^{2}}{r^{4}} \\
& =\frac{8 \cdot \pi \cdot G}{c^{4}}\left(p_{\perp}-p_{r}\right) .
\end{aligned}
$$

Our task is to explore the solutions of (8) and to obtain the fluid parameters $p_{r}, p_{\perp}$, and $\rho$ from (5), (6), and (7).

To solve the above equation we consider a seed solution as a particular case of Hajj-Boutros [26], Murad-Pant [27] and the electric intensity $E$ of the following form:

$$
\frac{E^{2}}{C}=\frac{q^{2}}{C r^{4}}=\frac{K C r^{2}\left(1+C r^{2}\right)^{-10 / 6}}{B^{2}}
$$

where $K$ is a positive constant defined as charge parameter. The electric intensity is so assumed that the model is physically significant and well behaved; that is, $E$ remains regular and positive throughout the sphere. In addition, $E$ vanishes at the center of the star and increases towards the boundary.

We also take

$$
\frac{8 \cdot \pi \cdot G}{c^{4}}\left(p_{\perp}-p_{r}\right)=\Delta=\frac{2 \cdot \alpha \cdot C^{2} r^{2}\left(1+C r^{2}\right)^{-10 / 6}}{B^{2}}
$$

where " $\Delta$ " is the anisotropy factor whose value is zero at the center and increases towards the boundary and " $\alpha$ " is a positive constant defined as anisotropy parameter. 


\section{Conditions for Well-Behaved Solution}

For well-behaved nature of the solution in isotropic coordinates, the following conditions should be satisfied (Mak and Harko [20] and Maurya and Gupta [22]).

(i) The solution should be free from physical and geometrical singularities, that is, finite and positive values of central pressure, central density, and nonzero positive values of $e^{\omega}$ and $e^{v}$.

(ii) The radial pressure $p_{r}$ must be vanishing, but the tangential pressure $p_{\perp}$ may not vanish at the boundary $r=r_{b}$ of the sphere. However, the radial pressure is equal to the tangential pressure at the centre of the fluid sphere.

(iii) The density $\rho$ and pressures $p_{r}, p_{\perp}$ should be positive inside the star.

(iv) $\left(d p_{r} / d r\right)_{r=0}=0$ and $\left(d^{2} p_{r} / d r^{2}\right)_{r=0}<0$ so that pressure gradient $d p_{r} / d r$ is negative for $0 \leq r \leq r_{b}$.

(v) $\left(d p_{\perp} / d r\right)_{r=0}=0$ and $\left(d^{2} p_{\perp} / d r^{2}\right)_{r=0}<0$ so that pressure gradient $d p_{\perp} / d r$ is negative for $0 \leq r \leq r_{b}$.

(vi) $(d \rho / d r)_{r=0}=0$ and $\left(d^{2} \rho / d r^{2}\right)_{r=0}<0$ so that density gradient $d \rho / d r$ is negative for $0 \leq r \leq r_{b}$.

Conditions (iv)-(vi) imply that pressure and density should be maximum at the centre and monotonically decreasing towards the surface.

(vii) Inside the static configuration the casualty condition should be obeyed; that is, the speed of sound should be less than the speed of light; that is, $0 \leq$ $\sqrt{d p_{r} / c^{2} d \rho}<1$ and $0 \leq \sqrt{d p_{\perp} / c^{2} d \rho}<1$. In addition to the above the velocity of sound should be decreasing towards the surface; that is, $(d / d r)\left(d p_{r} / d \rho\right)<0$ or $\left(d^{2} p_{r} / d \rho^{2}\right)>0$ and $(d / d r)\left(d p_{\perp} / d \rho\right)<0$ or $\left(d^{2} p_{\perp} / d \rho^{2}\right)>0$ for $0 \leq r \leq r_{b}$; that is, the velocity of sound is increasing with the increase of density. In this context it is worth mentioning that the equation of state at ultrahigh distribution has the property that the sound speed is decreasing outwards (Canuto and Lodenquai [28]).

(viii) A physically reasonable energy-momentum tensor has to obey the conditions $\rho \geq p_{r}+2 p_{\perp}$ and $\rho+p_{r}+$ $2 p_{\perp} \geq 0$.

(ix) The central red shift $Z_{0}$ and the surface red shift $Z_{b}$ should be positive and finite; that is, $Z_{0}=$ $\left[e^{-v / 2}-1\right]_{r=0}>0$ and $Z_{b}=\left[e^{-v_{b} / 2}-1\right]>0$ and both should be bounded.

(x) Electric intensity $E$, such that $E_{r=0}=0$ and is taken to be monotonically increasing.

(xi) The anisotropy factor $\Delta$ should be zero at the center and increasing towards the surface.

\section{A New Class of Solutions}

Equation (8) is solved by assuming the seed solution as a particular case of Hajj-Boutros [26], Murad-Pant [27] and the electric intensity $E$ and the anisotropy factor $\Delta$ in such a manner that the solution can be obtained and physically viable. Thus we have

$$
\begin{gathered}
e^{\omega / 2}=\mathbf{B}\left(1+C r^{2}\right)^{-1 / 6}, \quad x=C r^{2}, \quad y=\frac{d v}{d x} \\
q^{2}=\frac{K}{B^{2} C} x^{3}(1+x)^{-10 / 6}, \\
\frac{8 \cdot \pi \cdot G}{c^{4}}\left(p_{\perp}-p_{r}\right)=\Delta=\frac{2 \cdot \alpha \cdot C \cdot x \cdot(1+x)^{-10 / 6}}{B^{2}} .
\end{gathered}
$$

On substituting the above in (8), we get the following Riccatidifferential equation in $y$ :

$$
\frac{d y}{d x}+\frac{y}{3(1+x)}+\frac{y^{2}}{2}=\frac{36 K+36 \alpha-10}{36 \cdot(1+x)^{2}}
$$

which yields the following solution:

$$
e^{\nu / 2}=\frac{\left\{1+A\left(1+C r^{2}\right)^{2 S / 12}\right\}\left(1+C r^{2}\right)^{(4-S) / 12}}{\mathbf{B}^{2}},
$$

where $A, B, C$, and $K$ are arbitrary constants and

$$
S=\sqrt{72(K+\alpha)-4},
$$

where $S$ is real for $K+\alpha \geq 1 / 18$.

The expressions for density, radial pressure, and transversal pressure are given by

$$
\begin{aligned}
& \frac{8 \pi G \rho}{c^{2}}= \frac{C}{36 B^{2} f^{20}}\left(72+20 C r^{2}-36 K C r^{2}\right) \\
& \frac{8 \pi G p_{r}}{c^{4}}= \frac{C}{36 B^{2} f^{20}\left\{1+A f^{2 S}\right\}} \\
& \times\left[C r ^ { 2 } \left[A f^{2 S}(36 K+12+8 S)\right.\right. \\
&+(36 K+12-8 S)] \\
& \frac{8 \pi G p_{\perp}}{c^{4}=} \frac{C}{36 B^{2} f^{20}\left\{1+A f^{2 S}\right\}} \\
& \times\left[C r^{2}\left[A f^{2 S}(36 K+72 \alpha+12+8 S)+(2-S)\right]\right]
\end{aligned}
$$

where

$$
f=\left(1+C r^{2}\right)^{1 / 12} \text {. }
$$




\section{Properties of the New Solution}

The central values of pressure and density are given by

$$
\begin{aligned}
\left(\frac{8 \pi G p_{r}}{c^{4}}\right)_{r=0}= & \left(\frac{8 \pi G p_{\perp}}{c^{4}}\right)_{r=0} \\
= & \frac{C}{3 B^{2}(1+A)}[2(1+A)+S(A-1)] \\
& \left(\frac{8 \pi G \rho}{c^{2}}\right)_{r=0}=\frac{2 C}{B^{2}}
\end{aligned}
$$

The central values of pressure and density will be nonzero positive definite if the following conditions are satisfied:

$$
A>\frac{(S-2)}{(S+2)}, \quad C>0 .
$$

The pressure to density ratios are given by

$$
\begin{aligned}
\frac{p_{r}}{\rho c^{2}}=( & C r^{2}\left[f^{2 S} A(36 K+12+8 S)+(36 K+12-8 S)\right] \\
& \left.+12 \cdot\left[f^{2 S} A(2+S)+(2-S)\right]\right) \\
\times & \left(\left(1+A f^{2 S}\right)\left[72+20 C r^{2}-36 K C r^{2}\right]\right)^{-1} \\
\frac{p_{\perp}}{\rho c^{2}}= & \left(C r ^ { 2 } \left[f^{2 S} A(36 K+72 \alpha+12+8 S)\right.\right. \\
& +(36 K+72 \alpha+12-8 S)] \\
& \left.+12 \cdot\left[f^{2 S} A(2+S)+(2-S)\right]\right) \\
\times & \left(\left(1+A f^{2 S}\right)\left[72+20 C r^{2}-36 K C r^{2}\right]\right)^{-1} .
\end{aligned}
$$

Subjecting to the condition that the ratio of pressure to density should be positive and less than 1 at the centre, that is, $p_{0} / \rho_{0} c^{2} \leq 1$, the following inequality has to be satisfied.

$$
\begin{aligned}
\left(\frac{p_{r}}{\rho c^{2}}\right)_{r=0} & =\left(\frac{p_{\perp}}{\rho c^{2}}\right)_{r=0}=\frac{2(1+A)+S A-S}{6(1+A)} \\
& =\frac{1}{6}-\frac{S(1-A)}{6(1+A)} \leq 1 .
\end{aligned}
$$

All the values of $A$ which satisfy (21) will also lead to the condition $p_{0} / \rho_{0} c^{2} \leq 1$.
Differentiating (16) with respect to $r$, we get

$$
\begin{gathered}
\frac{8 \pi G}{c^{4}} \frac{d p_{r}}{d r}=\frac{C^{2} r}{216 B^{2}\left\{1+A f^{2 S}\right\}^{2} f^{32}} \\
\times\left[-8 \cdot C r^{2} \times\left[A^{2} f^{4 S}\{36 K+12+8 S\}\right.\right. \\
+\{36 K+12-8 S\} \\
\left.+2 A \cdot f^{2 S}\left(36 K+12-2 S^{2}\right)\right] \\
+12 \cdot\left[A^{2} f^{4 S}\{36 K-28-12 S\}\right. \\
+(36 K-28+12 S) \\
\left.\left.+2 A \cdot f^{2 S}\left(36 K-28+2 S^{2}\right)\right]\right] .
\end{gathered}
$$

Thus it is found that maxima of $p_{r}$ occur at the centre; that is,

$$
p_{r}^{\prime}=0 \Longrightarrow r=0, \quad \frac{8 \pi G}{c^{4}}\left(p_{r}^{\prime \prime}\right)_{r=0}=-v e .
$$

Thus the expression of the right-hand side of (26) is negative for all values of $A$ satisfying condition (21), showing thereby that the pressure $\left(p_{r}\right)$ is maximum at the centre and monotonically decreasing.

Differentiating (17) with respect to $r$, we get

$$
\begin{aligned}
& \frac{8 \pi G}{c^{4}} \frac{d p_{\perp}}{d r} \\
& =\frac{C^{2} r}{216 B^{2}\left\{1+A f^{2 S}\right\}^{2} f^{32}} \\
& \times\left[-8 \cdot C r^{2} \times\left[A^{2} f^{4 S}\{36 K+72 \alpha+12+8 S\}\right.\right. \\
& +\{36 K+72 \alpha+12-8 S\} \\
& \left.+2 A \cdot f^{2 S}\left(36 K+72 \alpha+12-2 S^{2}\right)\right] \\
& +12 \cdot\left[A^{2} f^{4 S}\{36 K+72 \alpha-28-12 S\}\right. \\
& +(36 K+72 \alpha-28+12 S) \\
& \left.\left.+2 A \cdot f^{2 S}\left(36 K+72 \alpha-28+2 S^{2}\right)\right]\right] .
\end{aligned}
$$

Thus it is found that maxima of $p_{\perp}$ occur at the centre; that is,

$$
p_{\perp}^{\prime}=0 \Longrightarrow r=0, \quad \frac{8 \pi G}{c^{4}}\left(p_{\perp}^{\prime \prime}\right)_{r=0}=-v e .
$$

Thus the expression of the right-hand side of (28) is negative for all values of $A$ satisfying condition (21), showing thereby that the transversal pressure is maximum at the centre and monotonically decreasing. 
Now differentiating equation (15) with respect to $r$ we get

$$
\begin{aligned}
& \frac{8 \pi G}{c^{2}} \frac{d \rho}{d r} \\
& \quad=\frac{C^{2} r}{216 B^{2} f^{32}}\left[-1200-432 K-160 \cdot C r^{2}+288 \cdot K C r^{2}\right] .
\end{aligned}
$$

Thus the maxima of $\rho$ occur at the centre; that is,

$$
\rho^{\prime}=0 \Longrightarrow r=0, \quad \frac{8 \pi G}{c^{2}}\left(\rho^{\prime \prime}\right)_{r=0}=\frac{(-1200-432 K) C^{2}}{216 B^{2}}
$$

Thus, the expression of the right-hand side of (30) is negative showing thereby that the density $\rho$ is maximum at the centre and monotonically decreasing.

The square of adiabatic sound speed at the centre, $\left(1 / c^{2}\right)(d p / d \rho)_{r=0}$, is given by

$$
\begin{aligned}
\frac{1}{c^{2}}\left(\frac{d p_{r}}{d \rho}\right)_{r=0} & \\
= & \frac{(36 K-28)(1+A)^{2}+12 S+4 A S^{2}-12 S A^{2}}{(-100-36 K)(1+A)^{2}}
\end{aligned}
$$

$<1$ and positive

$$
\begin{aligned}
\frac{1}{c^{2}}\left(\frac{d p_{\perp}}{d \rho}\right)_{r=0} & \\
= & \frac{(36 K+72 \alpha-28)(1+A)^{2}+12 S+4 A S^{2}-12 S A^{2}}{(-100-36 K)(1+A)^{2}}
\end{aligned}
$$

$<1$ and positive.

The causality condition is obeyed at the centre for all values of constants satisfying condition (21). Due to cumbersome expressions the trend of pressure-density ratios and adiabatic sound speeds are studied analytically after applying the boundary conditions.

\section{Boundary Conditions in Isotropic Coordinates}

For exploring the boundary conditions, we use the principle that the metric coefficients $g_{i j}$ and their first derivatives $g_{i j, k}$ in interior solution $(I)$ as well as in exterior solution $(E)$ are continuous up to and on the boundary $B$. The continuity of metric coefficients $g_{i j}$ of $I$ and $B$ on the boundary is the known first fundamental form. The continuity of derivatives of metric coefficients $g_{i j}$ of $I$ and $B$ on the boundary is the known second fundamental form.
The exterior field of a spherically symmetric static charged fluid distribution is described by Reissner-Nordstrom metric given by

$$
\begin{aligned}
d s^{2}= & \left(1-\frac{2 G M}{c^{2} R}+\frac{q^{2}}{R^{2}}\right) c^{2} d t^{2}-\left(1-\frac{2 G M}{c^{2} R}+\frac{q^{2}}{R^{2}}\right)^{-1} d R^{2} \\
& -R^{2}\left(d \theta^{2}+\sin ^{2} \theta d \phi^{2}\right)
\end{aligned}
$$

where $M$ is the mass of the ball as determined by the external observer and $R$ is the radial coordinate of the exterior region.

Since Reissner-Nordstrom metric (33) is considered as the exterior solution, we will arrive at the following conclusions by matching first and second fundamental forms:

$$
\begin{gathered}
e^{v_{b}}=\left[1-2 \frac{G M}{c^{2} R_{b}}+\frac{q_{b}^{2}}{R_{b}^{2}}\right] \\
R_{b}=r_{b} \cdot e^{\omega_{b} / 2}, \quad q_{\left(r=r_{b}\right)}=q_{b} \\
\frac{1}{2}\left(\omega^{\prime}+\frac{2}{r}\right)_{b} r_{b}=\left(1-2 \frac{G M}{c^{2} R_{b}}+\frac{q_{b}^{2}}{R_{b}^{2}}\right)^{1 / 2} \\
\frac{1}{2}\left(\nu^{\prime}\right)_{b} r_{b}=\left(\frac{G M}{c^{2} R_{b}}-\frac{q_{b}^{2}}{R_{b}^{2}}\right)\left(1-2 \frac{G M}{c^{2} R_{b}}+\frac{q_{b}^{2}}{R_{b}^{2}}\right)^{-1 / 2} .
\end{gathered}
$$

Equations (34) to (37) are four conditions, known as boundary conditions in isotropic coordinates. Moreover (35) and (37) are equivalent to zero pressure of the interior solution on the boundary.

Applying the boundary conditions from (34) to (37), we get the values of the arbitrary constants in terms of ReissnerNordstrom parameter " $d$," Schwarzschild parameters $u=$ $G M / c^{2} R_{b}$, and radius of the star $R_{b}$;

$$
\begin{gathered}
C=\frac{6(1-d)}{(6 d-4) r_{b}^{2}}>0 \text { for } u \leq \frac{10}{36}+\frac{q_{b}^{2}}{2 R_{b}^{2}} \\
A=\left(6\left(u-\frac{q_{b}^{2}}{R_{b}^{2}}\right) \cdot\left(1+C r_{b}^{2}\right)^{(4-S) / 12}-(4-S) \cdot C r_{b}^{2} \cdot d\right. \\
\left.\cdot\left(1+C r_{b}^{2}\right)^{(-8-S) / 12}\right) \\
\times\left((4+S) \cdot d \cdot C r_{b}^{2} \cdot\left(1+C r_{b}^{2}\right)^{(-8+S) / 12}\right. \\
\left.\quad-6\left(u-\frac{q_{b}^{2}}{R_{b}^{2}}\right)\left(1+C r_{b}^{2}\right)^{(4+S) / 12}\right)^{-1} \\
B=\sqrt{\frac{\left(1+C r_{b}^{2}\right)^{(4-S) / 12}+A\left(1+C r_{b}^{2}\right)^{(4+S) / 12}}{d}}
\end{gathered}
$$



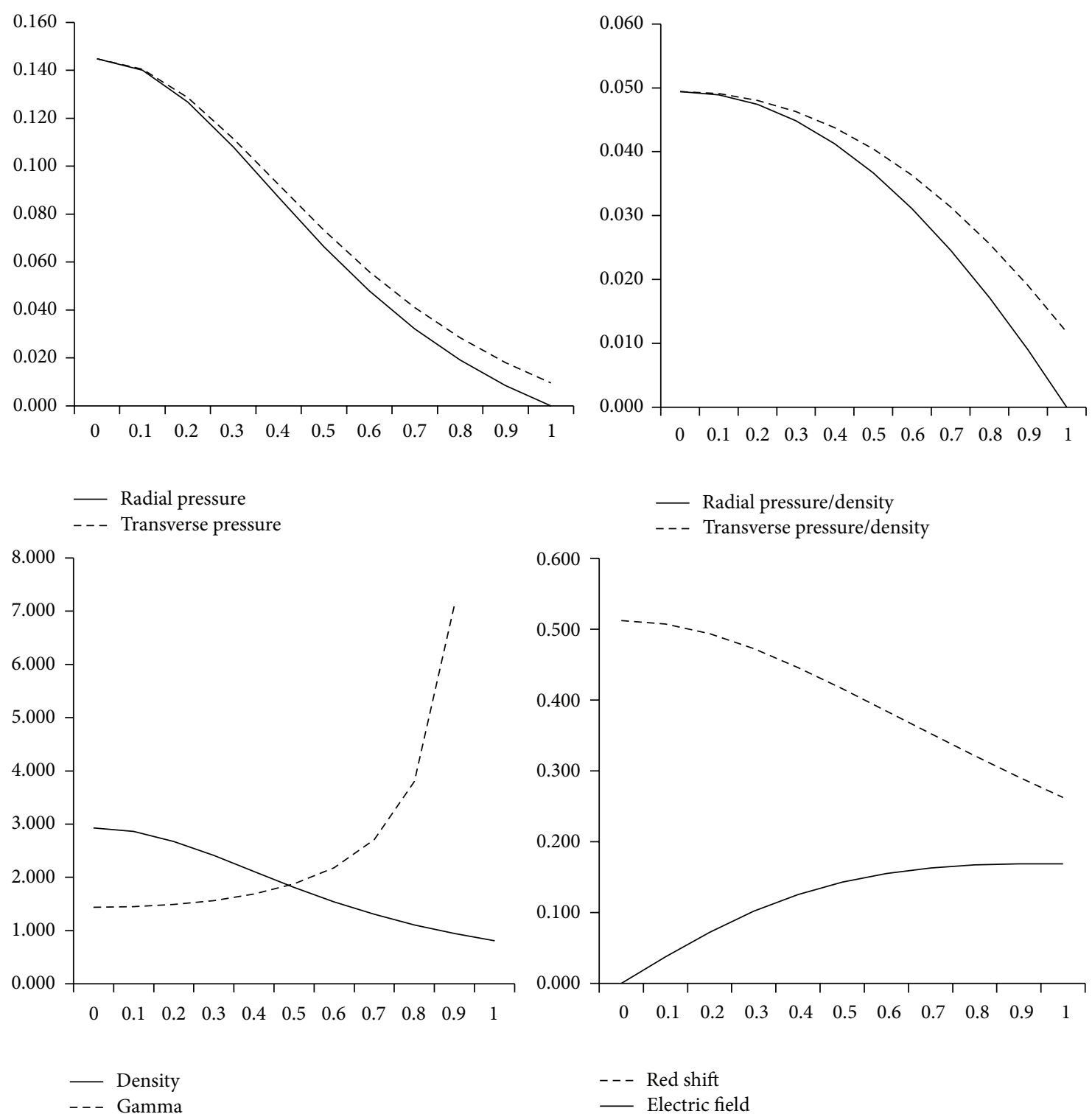

FIgURE 1: The variation of $p, \rho, p / \rho c^{2}, Z, \gamma$, and $E$ from centre to surface for $K=0.06$ and $\alpha=0.01$.

where we define a new parameter called Reissner-Nordstrom parameter " $d$ " given by

$$
d=\left[1-2 u+\frac{q_{b}^{2}}{R_{b}^{2}}\right]^{1 / 2}
$$

whose value lies within $2 / 3<d<1$ for $C r_{b}^{2}>0$.

Surface density is given by

$$
\frac{8 \pi G \rho}{c^{2}} R_{b}^{2}=\frac{C r_{b}^{2}}{36\left(1+C r_{b}^{2}\right)^{2}}\left[72+20 C r_{b}^{2}-36 K C r_{b}^{2}\right] .
$$

Central red shift is given by

$$
Z_{0}=\left[\frac{B^{2}}{1+A}-1\right]
$$

The surface red shift is given by

$$
Z_{b}=\left[e^{-v_{b} / 2}-1\right] .
$$

\section{Discussions and Conclusions}

From Figures 1 and 2 it has been observed that the physical quantities $p_{r}, p_{\perp}, p_{r} / \rho c^{2}, p_{\perp} / \rho c^{2}, d p_{r} / c^{2} d \rho, d p_{\perp} / c^{2} d \rho$, and $z$ are positive at the centre and within the limit of realistic state equation and monotonically decreasing while the quantities $\gamma, \Delta, E$ are increasing for all values of $K, \alpha$, and $u$ lying in the ranges $0.04 \leq K \leq 0.111,0<\alpha \leq 0.016$, and 

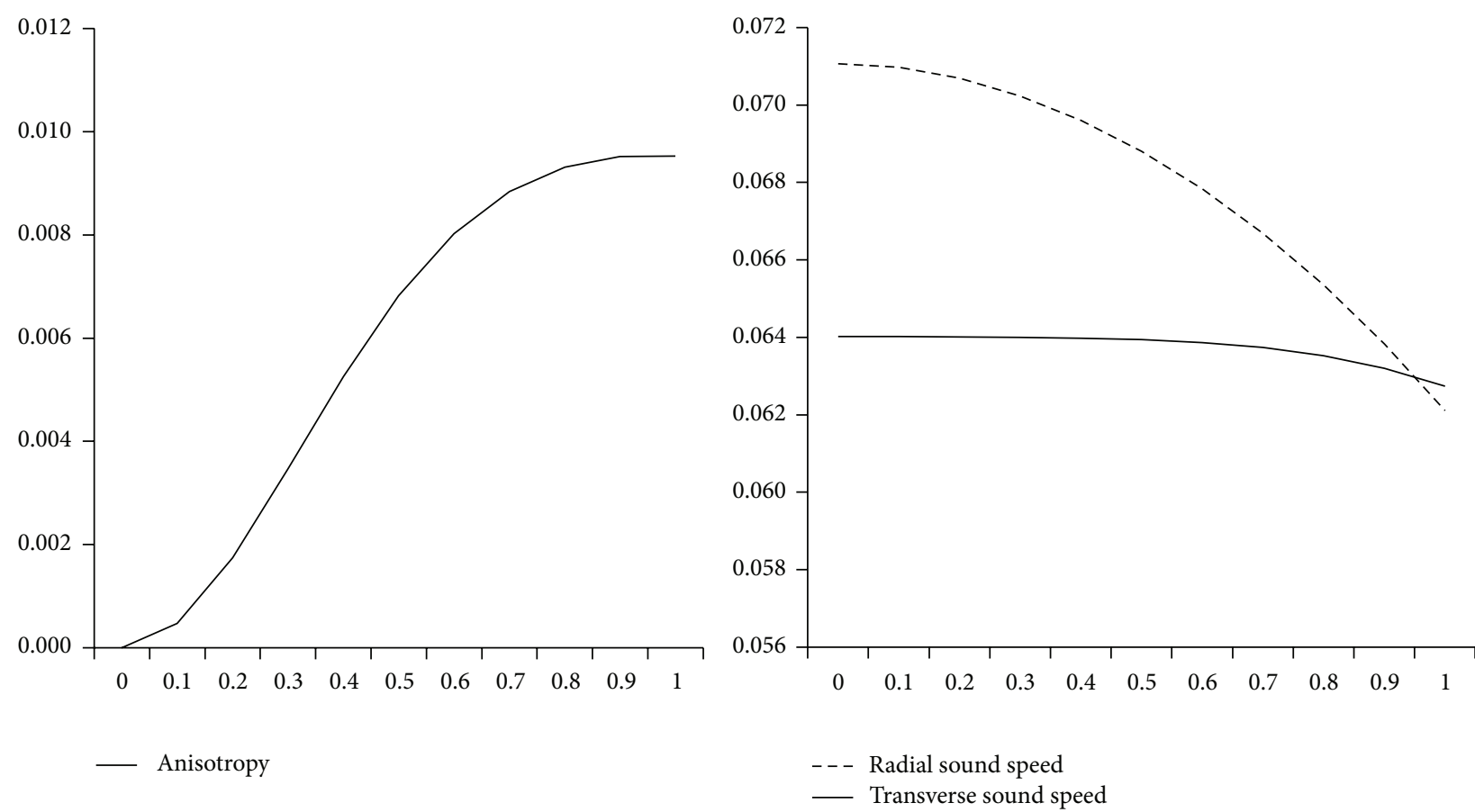

FigURE 2: The variation of $\left(1 / c^{2}\right)(d p / d \rho)$ and anisotropy from centre to surface for $K=0.06$ and $\alpha=0.01$.
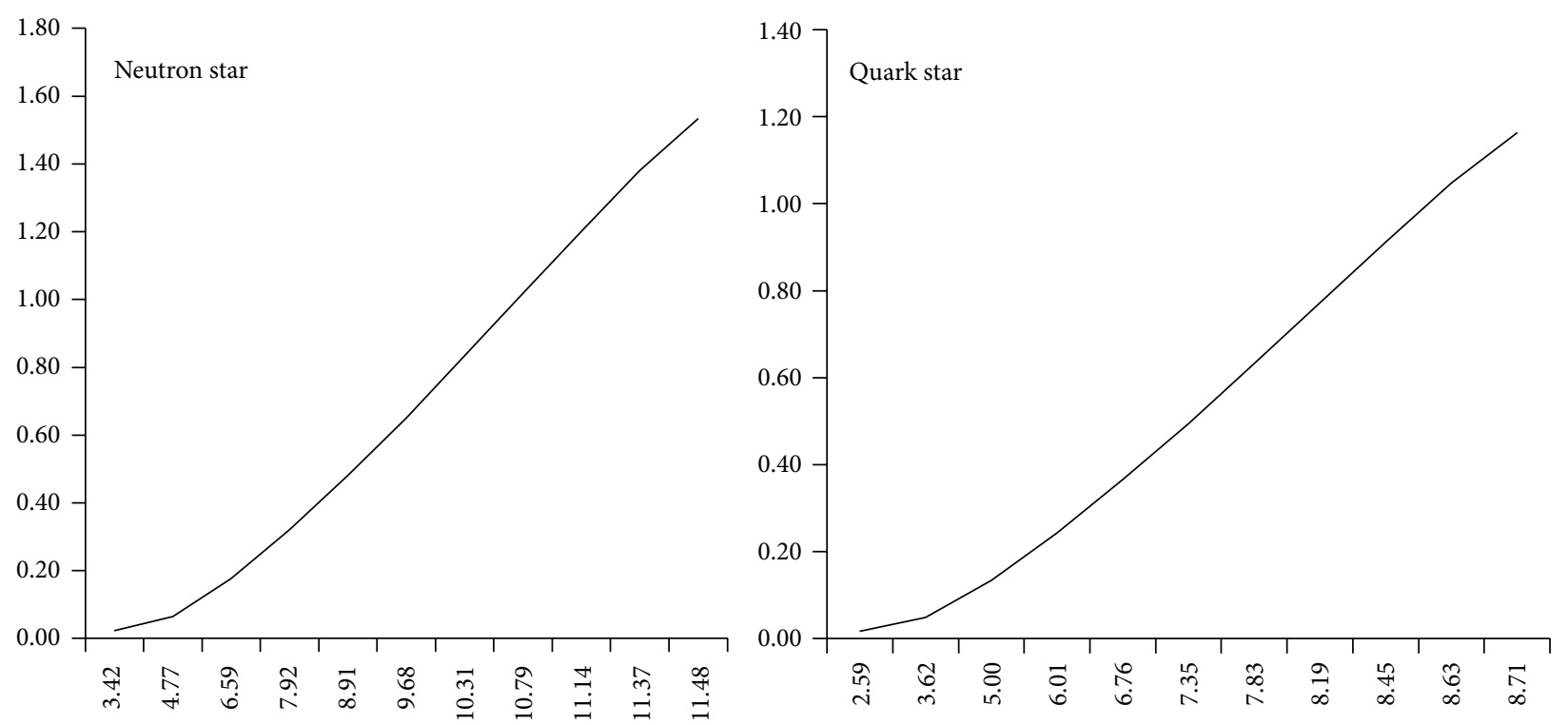

FIGURE 3: The variation of mass with radius of the superdense stars.

$0 \leq u \leq 0.2086$, respectively. For $K+\alpha>0.111$ the pressure is negative. With the increase in the value of $K$ from 0.04 to 0.111 the Schwarzschild parameter " $u$ " increases; hence the mass increases, but the value of $\alpha$ has to be decreased to 0 . With $\alpha=0$ we recover the isotropic model.

By increasing $K$ above 0.111 the causality condition is obeyed throughout within the ball, but the trend of adiabatic sound speed (transversal) is erratic. Thus, the solution is well behaved for all values of $u$ satisfying the inequality $0 \leq u \leq$
0.2086 for $K$ up to 0.111 and for $\alpha$ up to 0.016 . From Figure 3 it is clearly shown that the mass of the superdense star has a linear dependence on its radius.

In Tables 1 and 2 we present a model of superdense neutron star and quark star based on the particular solution discussed above. By assuming surface density, $\rho_{b}=4.6888 \times$ $10^{14} \mathrm{~g} / \mathrm{cm}^{3}$ corresponding to $\alpha=0.01, K=0.06$ for which $u=0.198$, the resulting well-behaved solution has a maximum mass $M=1.16 \mathrm{M}_{\odot}$ and radius $R=8.71 \mathrm{~km}$ 
TABLE 1: Variation of maximum mass of neutron star and corresponding radius $R_{b}$ with $u$ for $K=0.06$ and $\alpha=0.01$ by assuming the surface density $\rho_{b}=2.7 \times 10^{14} \mathrm{~g} / \mathrm{cm}^{3}$.

\begin{tabular}{lccc}
\hline$u$ & $\left(8 \pi G / C^{2}\right) \rho r_{b}^{2}$ & $R_{b}(\mathrm{~km})$ & $M / M_{\odot}$ \\
\hline 0.010 & 0.08605 & 3.41575 & 0.02299 \\
0.020 & 0.16661 & 4.77451 & 0.06413 \\
0.040 & 0.31173 & 6.59067 & 0.17622 \\
0.060 & 0.44198 & 7.92405 & 0.32153 \\
0.080 & 0.54874 & 8.91444 & 0.48155 \\
0.100 & 0.63497 & 9.68137 & 0.65060 \\
0.120 & 0.70596 & 10.31179 & 0.83478 \\
0.140 & 0.75730 & 10.78723 & 1.01872 \\
0.160 & 0.79117 & 11.13602 & 1.20163 \\
0.180 & 0.80834 & 11.36756 & 1.38018 \\
0.198 & 0.81007 & 11.47792 & 1.53364 \\
\hline
\end{tabular}

TABLE 2: Showing the variation of maximum mass of Quark star $\left(\rho_{b}=4.6888 \times 10^{14} \mathrm{~g} / \mathrm{cm}^{3}\right)$, its radius, surface red shift, surface electric intensity, and surface anisotropy with $u$ for $K=0.06$ and $\alpha=0.01$.

\begin{tabular}{lccccc}
\hline$u$ & $R_{B}(\mathrm{~km})$ & $M / M_{\odot}$ & $Z_{b}$ & $E_{b} r_{b}$ & $\Delta_{b} r_{b}^{2}$ \\
\hline 0.010 & 2.59201 & 0.01745 & 0.010101 & 0.008901 & 0.000026 \\
0.020 & 3.62309 & 0.04866 & 0.020408 & 0.017722 & 0.000105 \\
0.040 & 5.00127 & 0.13373 & 0.041667 & 0.035122 & 0.000411 \\
0.060 & 6.01310 & 0.24399 & 0.064963 & 0.053045 & 0.000938 \\
0.080 & 6.76465 & 0.36542 & 0.088850 & 0.070282 & 0.001646 \\
0.100 & 7.34663 & 0.49370 & 0.113586 & 0.087017 & 0.002524 \\
0.120 & 7.82502 & 0.63347 & 0.140901 & 0.104300 & 0.003626 \\
0.140 & 8.18579 & 0.77305 & 0.169317 & 0.121076 & 0.004886 \\
0.160 & 8.45047 & 0.91185 & 0.199472 & 0.137677 & 0.006318 \\
0.180 & 8.62618 & 1.04734 & 0.231679 & 0.154199 & 0.007926 \\
0.198 & 8.70992 & 1.16379 & 0.262626 & 0.169054 & 0.009526 \\
\hline
\end{tabular}

(for quark star) and by assuming the surface density $\rho_{b}=$ $2.7 \times 10^{14} \mathrm{~g} / \mathrm{cm}^{3}$ the obtained maximum mass is $M=1.53 \mathrm{M}_{\odot}$ and radius $R=11.48$ (for neutron star).

\section{Conflict of Interests}

The authors declare that there is no conflict of interests regarding the publication of this paper.

\section{Acknowledgments}

The authors acknowledge their gratitude to Major General Ashok Ambre, SM, Deputy Commandant, NDA, for his motivation and encouragement. They also extend their gratitude to Professor O. P. Shukla, Principal, NDA, for his encouragement. They are grateful to the anonymous referees for their rigorous review, constructive comments, and useful suggestions.

\section{References}

[1] B. V. Ivanov, "Static charged perfect fluid spheres in general relativity," Physical Review D, vol. 65, no. 10, Article ID 104001, 17 pages, 2002.

[2] F. de Felice, Y. Yu, and J. Fang, "Relativistic charged spheres," Monthly Notices of the Royal Astronomical Society, vol. 277, p. L17, 1995.

[3] W. B. Bonnor, "The equilibrium of charged sphere," Monthly Notices of the Royal Astronomical Society, vol. 137, no. 3, pp. 239251, 1965.

[4] N. Bijalwan, "Exact solutions: classical electron model," Astrophysics and Space Science, vol. 336, no. 2, pp. 485-489, 2011.

[5] T. E. Kiess, "A nonsingular perfect fluid classical lepton model of arbitrarily small radius," International Journal of Modern Physics D, vol. 22, Article ID 1350088, 2013.

[6] S. D. Maharaj and P. M. Takisa, "Regular models with quadratic equation of state," General Relativity and Gravitation, vol. 44, no. 6, pp. 1419-1432, 2012.

[7] R. Ruderman, "Pulsars: structure and dynamics," Annual Review of Astronomy and Astrophysics, vol. 10, pp. 427-476, 1972.

[8] R. Sharma and S. D. Maharaj, "A class of relativistic stars with a linear equation of state," Monthly Notices of the Royal Astronomical Society, vol. 375, pp. 1265-1268, 2007.

[9] B. V. Ivanov, "Collapsing shear-free perfect fluid spheres with heat flow," General Relativity and Gravitation, vol. 44, no. 7, pp. 1835-1855, 2012.

[10] A. V. Astashenok, S. Capozziello, and S. D. Odintsov, "Further stable neutron star models from $\mathrm{f}(\mathrm{R})$ gravity," 2013, http://arxiv .org/abs/1309.1978.

[11] S. Fatema and M. H. Murad, "An exact family of EinsteinMaxwell Wyman-Adler solution in general relativity," International Journal of Theoretical Physics, vol. 52, no. 7, pp. 2508-2529, 2013.

[12] J. L. Zdunik, "Strange stars-linear approximation of the EOS and maximum QPO frequenc," Astronomy \& Astrophysics, vol. 359, pp. 311-315, 2000.

[13] H. Dong, T. T. S. Kuo, H. K. Lee, R. Machleidt, and M. Rho, "Half-Skyrmions and the equation of state for compact-star matter," Physical Review C, vol. 87, Article ID 054332, 2013.

[14] K. Dev and M. Gleiser, "Anisotropic stars: exact solutions," General Relativity and Gravitation, vol. 34, no. 11, pp. 1793-1818, 2002.

[15] K. Komathiraj and S. D. Maharaj, "Tikekar superdense stars in electric fields," Journal of Mathematical Physics, vol. 48, Article ID 042501, 12 pages, 2007.

[16] K. Komathiraj and S. D. Maharaj, "Analytical models for quark stars," International Journal of Modern Physics D, vol. 16, no. 11, pp. 1803-1811, 2007.

[17] S. Thirukkanesh and F. C. Ragel, "Exact anisotropic sphere with polytropic equation of state," Pramana, vol. 78, no. 5, pp. 687696, 2012.

[18] P. M. Takisa and S. D. Maharaj, "Compact models with regular charge distributions," Astrophysics and Space Science, vol. 343, no. 2, pp. 569-577, 2013.

[19] P. M. Takisa and S. D. Maharaj, "Some charged polytropic models," General Relativity and Gravitation, vol. 45, no. 10, pp. 1951-1969, 2013.

[20] M. K. Mak and T. Harko, "An exact anisotropic quark star model," Chinese Journal of Astronomy and Astrophysics, vol. 2, no. 3, pp. 248-260, 2002. 
[21] M. K. Mak, P. N. Dobson, and T. Harko, "Exact model for anisotropic relativistic stars," International Journal of Modern Physics D, vol. 11, pp. 207-221, 2002.

[22] S. K. Maurya and Y. K. Gupta, "A family of anisotropic superdense star models using a space-time describing charged perfect fluid distributions," Physica Scripta, vol. 86, Article ID 025009, 2012.

[23] S. K. Maurya and Y. K. Gupta, "Charged fluid to anisotropic fluid distribution in general relativity," Astrophysics and Space Science, vol. 344, no. 1, pp. 243-251, 2013.

[24] M. Chaisi and S. D. Maharaj, "Anisotropic static solutions in modelling highly compact bodies," Journal of Physics, vol. 66, no. 3, pp. 609-614, 2006.

[25] T. Feroze and A. A. Siddiqui, "Charged anisotropic matter with quadratic equation of state," General Relativity and Gravitation, vol. 43, no. 4, pp. 1025-1035, 2011.

[26] J. Hajj-Boutros, "Spherically symmetric perfect fluid solutions in isotropic coordinates," Journal of Mathematical Physics, vol. 27, no. 5, pp. 1363-1366, 1986.

[27] H. M. Murad and N. Pant, "A class of exact isotropic solutions of Einstein's equations and relativistic stellar models in general relativity," Astrophysics and Space Science, vol. 350, no. 1, pp. 349-359, 2014.

[28] V. Canuto and J. Lodenquai, "Solidification of neutron matter," Physical Review C, vol. 12, no. 6, pp. 2033-2037, 1975. 

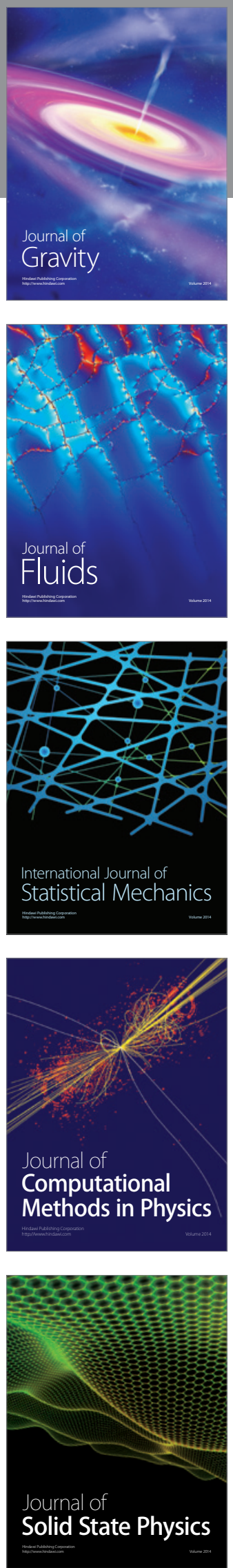

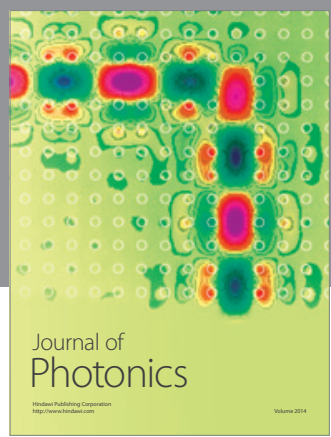

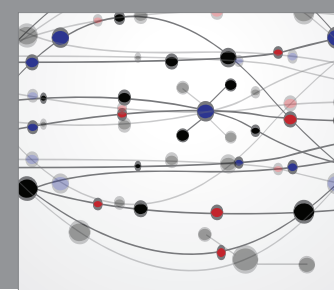

The Scientific World Journal

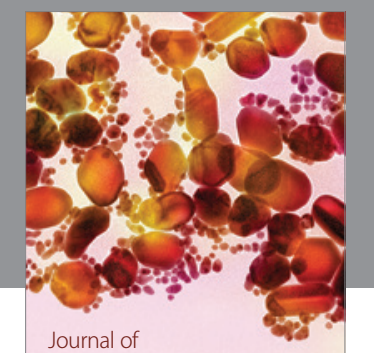

Soft Matter
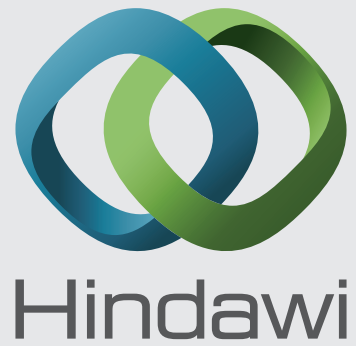

Submit your manuscripts at

http://www.hindawi.com
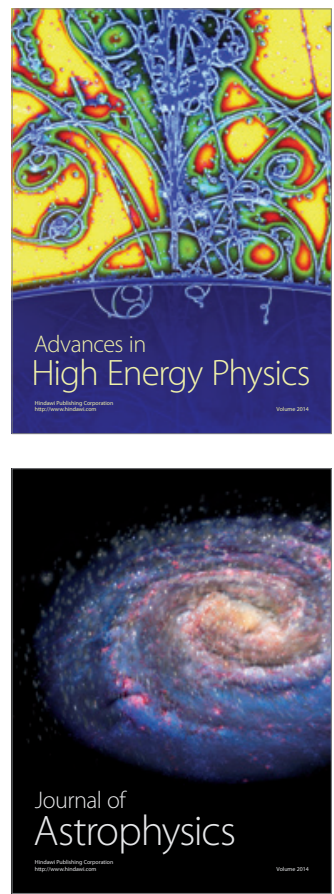
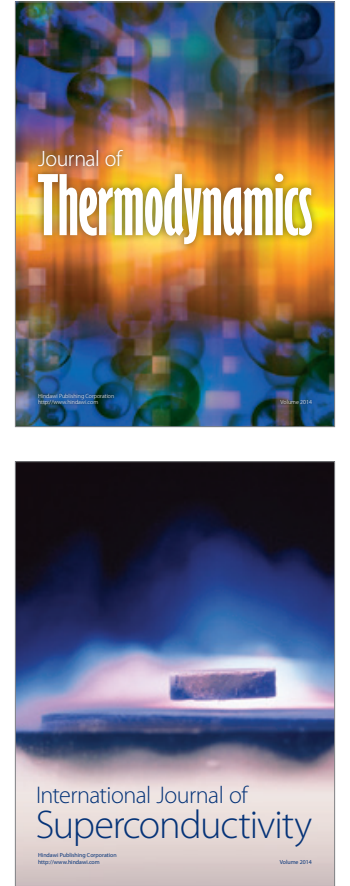
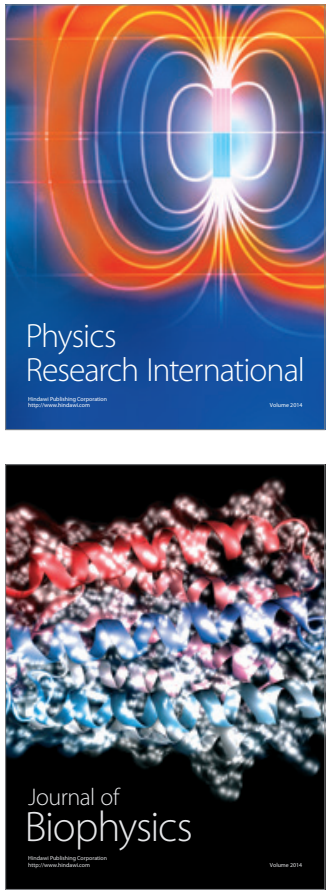
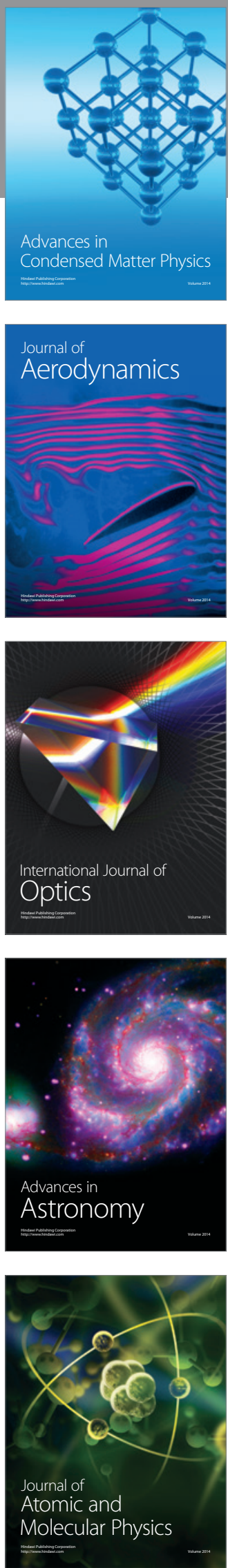